\title{
Annual fractional exhaled nitric oxide measurements and exacerbations in severe asthma: a multicenter observational cohort study
}

Yuki Abe ( $\square$ abeyuki@pop.med.hokudai.ac.jp )

Hokkaido Daigaku

Masaru Suzuki

Hokkaido University

Hirokazu Kimura

Hokkaido University

Kaoruko Shimizu

Hokkaido University

Hironi Makita

Hokkaido University

Masaharu Nishimura

Hokkaido University

Satoshi Konno

Hokkaido University

\section{Research}

Keywords: asthma, biomarker, coefficient of variation, exacerbation, fractional exhaled nitric oxide, longitudinal assessment, variability

Posted Date: July 22nd, 2020

DOI: https://doi.org/10.21203/rs.3.rs-40852/v2

License: (9) (i) This work is licensed under a Creative Commons Attribution 4.0 International License. Read Full License 


\section{Abstract}

Background: Fractional exhaled nitric oxide (FeNO) reflects eosinophilic inflammation of the airways. However, the significance of longitudinal assessment of FeNO, including its variability, in the clinical course of severe asthma remains unclear. The aim of this study is to examine the association between long-term changes in FeNO and the development of exacerbations in severe asthma.

Methods: Among the severe asthma patients enrolled in the Hokkaido Severe Asthma Cohort Study, 100 patients with severe asthma who completed a 3-year follow-up in which FeNO was measured annually were included. According to the FeNO level at baseline, 1 year, and 2 years, the patients were classified into three groups: the sustained high group ( $\geq 50 \mathrm{ppb}$ at all three visits), the sustained low group ( $<25 \mathrm{ppb}$ at all three visits), and the intermediate group (other). Subjects in the intermediate group were further classified into two groups based on the median value of the coefficient of variation (CV) of FeNO during the 3 years (high CV and low CV intermediate groups).

Results: The sustained high group experienced shorter exacerbation-free survival and more frequent exacerbations than the sustained low group (median number of exacerbation events, 3 vs. $0, p=0.01$ ). In the intermediate group, the high CV group experienced shorter exacerbation-free survival than the low CV group, and the CV of FeNO was an independent contributing factor to the development of exacerbations.

Conclusion: Persistence of FeNO above 50 ppb over the years as well as the presence of large variations in FeNO levels were associated with the development of exacerbations in patients with severe asthma.

\section{Introduction}

Some patients with severe asthma remain uncontrolled despite good adherence to high-dose inhaled corticosteroids (ICS) and treatment of contributory factors; severe asthma also places a major health and economic burden ${ }^{1}$. Severe asthma is heterogeneous, and distinct phenotypes have been recognized even in cigarette smokers with severe asthma ${ }^{2}$. On the other hand, non-invasive measurement of fractional exhaled nitric oxide (FeNO) reflects eosinophilic inflammation in the lower respiratory tract ${ }^{3}$. Nitric oxide is generated in the airways by inducible nitric oxide synthase, which is produced by type 2 cytokines, such as interleukin (IL)-4 and IL-134. FeNO is associated with the levels of eosinophils in the airway mucosa, bronchoalveolar lavage fluid, sputum, and blood ${ }^{5,6}$; thus, FeNO levels are high in patients with asthma characterized by type 2 inflammation ${ }^{7}$. High FeNO levels have also been reported to be associated with good short-term response to ICS ${ }^{8,9}$. We previously reported that in patients with severe asthma, FeNO at baseline had a significant potential to predict future exacerbations ${ }^{10}$. However, the significance of longitudinal assessment of FeNO, including its variability, in the clinical course of severe asthma, especially the development of exacerbations, remains unclear.

The Hokkaido Severe Asthma Cohort Study or the Hokkaido-based Investigative Cohort Analysis for Refractory Asthma (Hi-CARAT) is a multicenter observational cohort study that primarily aimed to 
characterize patients with severe asthma, including smokers. We completed a 3-year follow-up with annual assessments of FeNO and other clinical parameters. In this study, we examined the association of long-term changes in FeNO levels in a 3-year observational cohort of patients with severe asthma and the development of exacerbations.

\section{Methods}

\section{Study protocol and Participants}

The study is based on data from the Hokkaido Severe Asthma Cohort Study (Hi-CARAT), which has previously been described in detail ${ }^{10,11}$. Patients with severe asthma were enrolled from Hokkaido University Hospital (Sapporo, Japan) and 29 affiliated hospitals and clinics between February 2010 and September 2012. This study was approved by the ethics committees of all hospitals, and all subjects provided written informed consent. This study was registered in the University Hospital Medical Information Network Center Clinical Trials Registry system (https://upload.umin.ac.jp/cgi-openbin/ctr/ctr_view.cgi?recptno=R000003917). The diagnosis of severe asthma is based on the American

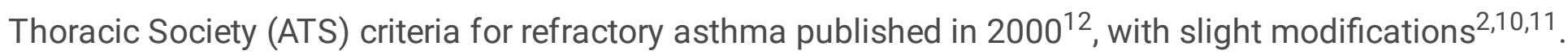
Participants stayed at Hokkaido University Hospital for 2 days for initial screening and were followed up every 1 year on an outpatient basis for 3 years. Patients who were well-controlled under current medications were asked if within 1 year they experienced episodic deterioration of symptoms, urgent care visits, and rescue use of short-acting bronchodilators when the dose of the current medication was reduced. During the study period, anti-IL 5 or anti-IL-4R antibody was not administered to any subject because of its clinical unavailability in Japan. We excluded subjects who had poor adherence to their medications and inadequate medication inhalation technique upon their initial visit ${ }^{11}$. Subjects with low adherence $(<70 \%)$ were excluded from the final analysis.

\section{FeNO measurement}

FeNO levels were measured using the single-breath online method with NIOX MINO (Aerocrin, Stockholm, Sweden) according to the ATS guidelines ${ }^{8}$. FeNO levels were measured four times: at the initial screening at visit entry (VE), 1 year later (V1), 2 years later (V2), and 3 years after VE (V3). Detailed measurements of other biomarkers have been described in detail in previous reports $2,10,11$.

\section{Assessment of exacerbation}

Asthma exacerbation was defined as the need for systemic corticosteroids for more than 3 days and/or hospital admission, corresponding to "severe exacerbation" based on the European Respiratory Society/ATS guidelines for severe asthma ${ }^{13}$.

Statistical analysis 
Descriptive statistics of patient characteristics within the cohort were calculated. Continuous variables were summarized using mean and standard deviation for continuous parametric variables, and median and interquartile range (difference between the 25th and 75th percentiles) for continuous non-parametric variables. To examine the differences among groups, we used the Chi-square test for categorical variables, the student's $t$-test or one-way analysis of variance for continuous parametric variables, and the Mann-Whitney $U$ test or Kruskal-Wallis test for continuous non-parametric variables. Post-hoc Dunn's test was used for non-parametric multiple comparisons. Factors associated with exacerbation-free survival were analyzed using the Cox proportional hazards model and the Kaplan-Meier method with the log-rank test. Factors associated with the frequency of exacerbation were analyzed using the Poisson regression model. Results were presented as hazard ratios and $95 \%$ confidence intervals (Cls). For cluster analysis, a hierarchical clustering method was performed. Statistical significance was defined as $p<0.05$. Statistical analyses were performed using JMP (SAS Institute Inc., Cary, NC, USA) and EZR version 1.42 (Saitama Medical Center, Jichi Medical University, Saitama, Japan), which is a graphical user interface for R software (The R Foundation for Statistical Computing, Vienna, Austria) ${ }^{14}$.

\section{Results}

Demographic characteristics of the subjects

A flow chart demonstrating the study process from the initial screening at visit entry (VE) to 1 year later (V1), 2 years later (V2), and 3 years after VE in the follow-up period (V3) is shown in Figure 1. Altogether, 100 subjects with severe asthma whose FeNO levels were measured at all visits of VE, V1, and V2 were analyzed in this study. The characteristics of the 100 subjects are shown in Table 1 . The average age at registration was $58.5 \pm 12.2$ years; $58 \%$ of the patients were male $(n=58)$ and $61 \%$ were atopic $(n=61)$;

$12(12 \%)$ of the subjects were current smokers and 51 (51\%) were ex-smokers; 37 subjects ( $37 \%)$ used daily oral corticosteroids (OCS). The median FeNO at baseline (VE) was $27.5 \mathrm{ppb}$ (range, 17.0-47.5 ppb). During the 3-year follow-up period, 59 subjects (59\%) experienced at least one exacerbation, and the median number of exacerbation events was 1.5 (interquartile range, $0-4$ ).

\section{Annual changes in FeNO}

We used FeNO values at $25 \mathrm{ppb}$ and $50 \mathrm{ppb}$ as thresholds indicating low and high levels of FeNO based on the ATS guidelines ${ }^{8}$. As shown in Figure 2, 24\% of the subjects had FeNO levels of $50 \mathrm{ppb}$ or greater at baseline (VE); $50 \%$ of them still had FeNO level of at least 50 ppb after 1 year (V1), and $75 \%$ of them had sustained FeNO level of at least 50 ppb after 2 years (V2). On the other hand, $47 \%$ of the subjects had FeNO levels less than $25 \mathrm{ppb}$ at baseline. Approximately $85 \%$ of them still had FeNO levels lower than 25 ppb after 1 year, and $82 \%$ of them had FeNO less than 25 ppb after 2 years.

We classified the subjects into three groups according to FeNO values at VE, V1, and V2: (1) the sustained high group ( $\geq 50 \mathrm{ppb}$ at all three visits), (2) the sustained low group ( $<25 \mathrm{ppb}$ at all three visits), and (3) the intermediate group, which did not meet any of the two aforementioned criteria. Nine (9\%) subjects 
were classified into the sustained high group, 33 (33\%) into the sustained low group, and 58 (58\%), intermediate group. After 3 years (V3), $88 \%$ of the sustained high group had FeNO $\geq 50 \mathrm{ppb}$ and $83 \%$ of the sustained low group had FeNO $<25$ ppb (Figure 2).

As shown in Table 1, the use of regular OCS was frequent in the sustained high group, and the prevalence of sinusitis was low in the sustained low group. During the follow-up period, exacerbation events were more frequent in the sustained high group than in the sustained low group (median number of exacerbation events, 3 vs. $0, p=0.01$, Figure 3 ).

Analysis of the intermediate group

We calculated the individual coefficients of variation (CV) of FeNO levels from VE to V3 for subjects in the intermediate group (median, 0.335 ; range, $0.200-0.520$ ) and classified them into two groups (high CV and low CV intermediate groups, $\mathrm{n}=29$ each) based on the median value. Table 2 shows the clinical characteristics of the two groups. There were no significant differences in age, body mass index, or pulmonary function test results between the two groups. The asthma control test score, asthma quality of life questionnaire score, and score for sinusitis (Lund-Mackay score) also did not differ between the two groups. The smoking and depression scores (Self-Rating Depression Scale) were significantly higher in the high CV intermediate group than in the latter. The daily dose of ICS tended to be higher in the high CV intermediate group, although this did not reach statistical significance. Moreover, exacerbation-free survival was shorter in the high CV intermediate group than in the low CV intermediate group (Figure 4). The Kaplan-Meier curve for exacerbation-free survival of the high CV intermediate group was similar to that of the sustained high group, and the Kaplan-Meier curve of the low CV intermediate group was similar to that of the sustained low group ( $p=0.03$, log-rank test among the four groups) (Figure 4$)$. When subjects in the high CV intermediate group were classified into two groups based on the pattern of FeNO variation by cluster analysis (Figure 5), there was no difference in exacerbation-free survival among the clustered groups; most of the subjects in the high CV intermediate group experienced their first exacerbation within 1 year (Figure 6).

In the intermediate group, the multivariate Cox proportional hazards model showed that the CV of FeNO was a significant and independent risk factor for the early onset of the first exacerbation event (Table 3). Furthermore, the multivariate Poisson regression model showed that blood eosinophil count and CV of FeNO were significantly associated with exacerbation frequency during the follow-up period (Table 3).

\section{Discussion}

We examined the association of long-term changes in FeNO with the development of exacerbation in severe asthma using data from the Hokkaido Severe Asthma Cohort Study (Hi-CARAT), a prospective cohort study. We found that persistence of FeNO levels above $50 \mathrm{ppb}$ over the years was associated with the development of exacerbations in patients with severe asthma. In addition, even if the FeNO level was not sustainably above $50 \mathrm{ppb}$, the large variation in FeNO levels was associated with the development of exacerbations. 
FeNO has been found to reflect eosinophilic inflammation in the airways of asthmatic patients ${ }^{8}$. Previous studies have also supported FeNO's role in reflecting asthma control ${ }^{15-17}$ and as a biomarker of response to ICS therapy ${ }^{18,19}$. On the other hand, eosinophilic airway inflammation measured based on induced sputum ${ }^{20}$ and $\mathrm{FeNO}^{21}$ in asthmatic patients is known to exhibit temporal oscillations. Therapeutic strategies targeting type 2 inflammation in asthmatic patients may be beneficial if they stabilize both the amplitude and variability of eosinophilic airway inflammation over time; therefore, longitudinal measurement of FeNO is important for monitoring the inflammatory status of the asthmatic airways.

We previously reported the presence of a phenotype of severe asthma with frequent exacerbations and that FeNO levels at baseline are significantly associated with exacerbation status during the 3-year follow-up period among patients with several type 2 biomarkers ${ }^{10}$. In the present study, persistently high FeNO levels were clearly associated with the development of exacerbations, consistent with our previous findings. On the other hand, annual FeNO levels in the intermediate group were highly variable despite the patients receiving high-dose ICS therapy. Intraday or daily variation in FeNO has been reported to provide useful information about asthma control and the risk of future exacerbations ${ }^{22}$. However, few studies have evaluated the annual changes in FeNO. The present study showed for the first time that FeNO levels could fluctuate annually in patients with severe asthma.

In healthy individuals, Ekroos et al. reported that a change of $30-35 \%$ or more in FeNO levels within the interval of 1 to 3 weeks should be considered abnormal and proposed this value as a cutoff for significant variation in FeNO level ${ }^{23}$. De Laurentiis et al. investigated the variation in FeNO level over 1 year in patients with chronic obstructive pulmonary disease (COPD), and reported that the CV of FeNO was significantly associated with exacerbation rate, but not with baseline FeNO levels and degree of airflow limitation ${ }^{24}$. In this study, the cutoff for FeNO variability was set at $40 \%$ based on the upper limit of the $95 \% \mathrm{Cl}$ of the mean of the entire COPD group. In another study on long-term variation in FeNO in lung transplant recipients, Antus et al. reported that a variation in FeNO levels of more than $30 \%$ during 2 years of follow-up was a specific marker of acute infection ${ }^{25}$. Furthermore, Stern et al. investigated daily FeNO values over 192 days in atopic asthmatic children and found that fluctuations in FeNO values were correlated over time scales of more than a month (long-range correlation). They also revealed that the day-by-day cross-correlation of FeNO values and symptom scores was stronger in subjects with moderate or severe exacerbations ${ }^{26}$. In the present study, we found that high variability in annual FeNO was associated with the development of exacerbations in the subset (intermediate group) of adults with severe asthma. Taken together, large variability in FeNO levels within a relatively long-term period appears to be associated with exacerbation events in lung diseases. Such variation in FeNO levels may reflect the instability and reduced adaptive capacity of the airways to changing environmental conditions ${ }^{27}$.

ICS or OCS treatment is a major factor in reducing FeNO levels as well as its long-term variability ${ }^{18,28}$. This issue may not largely influence our findings because all subjects received high-dose ICS with good adherence and $37 \%$ of the subjects were on systemic corticosteroids. Indeed, $77.8 \%$ of subjects in the sustained FeNO high group were on daily OCS treatment, and the high CV intermediate group tended to 
take higher amounts of ICS than the low CV intermediate group. Anti-IL4R antibody has also been shown to reduce FeNO levels, but anti-IL-4R antibody was not administered to the subjects because of its clinical unavailability in Japan during the study period. Therefore, the FeNO levels in the present study reflect the pathological status of severe asthma despite intensive treatment with corticosteroids. Persistence of high FeNO levels and high variability would be a good indication to initiate treatment with biologics in patients with severe asthma.

This study has several limitations. First, the sample size may have been small, despite recruitment of subjects from 29 affiliated hospitals/pulmonary clinics. However, we intentionally focused on severe asthmatics who had been managed by respiratory physicians, and all subjects were carefully followed with a low dropout rate. Second, FeNO level was measured only once per year, which made it difficult to determine the causal relationship between variation in FeNO levels and asthma exacerbation. As discussed above, FeNO can fluctuate on an intraday or daily basis. However, such frequent FeNO measurement is not realistic in current clinical practice, and our findings suggest that even annual FeNO measurement would be helpful for decision-making in the management of severe asthma patients.

\section{Conclusions}

Persistence of FeNO level above 50 ppb over the years and the presence of large variations in FeNO levels were associated with the development of exacerbations in patients with severe asthma. The findings of the present study emphasize the importance of longitudinal assessment of FeNO levels for the management of severe asthma. Further studies on the mechanism of the variation in FeNO levels and its association with exacerbations are required in the future.

\section{Declarations}

\section{Ethics approval and consent to participate}

This study was approved by the ethics committees of all hospitals, and all subjects provided written informed consent. This study was registered in the University Hospital Medical Information Network Center Clinical Trials Registry system (https://upload.umin.ac.jp/cgi-open-bin/ctr/ctr_view.cgi? recptno=R000003917).

\section{Consent for publication}

Not applicable.

\section{Availability of data and materials}

The datasets used and/or analysed during the current study are available from the corresponding author on reasonable request.

\section{Competing interests}


The authors YA, HK, KS, HM, and SK declare no conflicts of interest. MS received grants from GlaxoSmithKline, AstraZeneca, and Novartis outside the submitted work. MN received grants from the Japan Allergy Foundation, Astra Zeneca, and Kyorin during the conduct of the study.

\section{Funding}

The Hokkaido Severe Asthma Cohort Study or the Hokkaido-based Investigative Cohort Analysis for Refractory Asthma (Hi-CARAT) is supported by the Ministry of Education, Culture, Sports, Science and Technology of Japan (24249049 to MN, 26461151 to SK) and a research grant from the Japan Allergy Foundation, AstraZeneca, and Kyorin.

\section{Authors' contributions}

YA contributed to acquisition and interpretation of data, statistical analysis, and drafting the manuscript. $\mathrm{HK}, \mathrm{KS}$, and HM contributed to acquisition and interpretation of data. MS, MN, and KS contributed to study concept and design, acquisition and interpretation of data, and finalizing the manuscript. All authors read and approved the final manuscript.

\section{Acknowledgements}

The authors thank Professor Elizabeth Juniper for approving the use of the Asthma Quality of Life Questionnaire; Hideka Ashikaga, Ayako Kondo, and Yuko Takagi of the Central Office of Hokkaido Medical Research Institute for Respiratory Disease; and the staff of Exam Co., Ltd. for their assistance in completing this work.

\section{Abbreviations}

ICS, inhaled corticosteroids

FeNO, fractional exhaled nitric oxide

IL, interleukin

Hi-CARAT, Hokkaido-based Investigative Cohort Analysis for Refractory Asthma

ATS, American Thoracic Society

$\mathrm{Cl}$, confidence interval

OCS, oral corticosteroids

$\mathrm{CV}$, coefficient of variation

\section{References}


1. Global Initiative for Asthma. Global Strategy for Asthma Management and Prevention (2020 update). Available at: https://ginasthma.org/wp-content/uploads/2020/06/GINA-2020-report_20_06_04-1wms.pdf. Accessed June 15, 2020.

2. Konno S, Taniguchi N, Makita H, Nakamaru Y, Shimizu K, Shijubo N, et al. Distinct Phenotypes of Smokers with Fixed Airflow Limitation Identified by Cluster Analysis of Severe Asthma. Ann Am Thorac Soc 2018;15:33-41.

3. ATS/ERS recommendations for standardized procedures for the online and offline measurement of exhaled lower respiratory nitric oxide and nasal nitric oxide, 2005. Am J Respir Crit Care Med 2005;171:912-30.

4. Ray A, Oriss TB, Wenzel SE. Emerging molecular phenotypes of asthma. Am J Physiol Lung Cell Mol Physiol 2015;308:L130-40.

5. Silkoff PE, Lent AM, Busacker AA, Katial RK, Balzar S, Strand M, et al. Exhaled nitric oxide identifies the persistent eosinophilic phenotype in severe refractory asthma. J Allergy Clin Immunol 2005;116:1249-55.

6. Korevaar DA, Westerhof GA, Wang J, Cohen JF, Spijker R, Sterk PJ, et al. Diagnostic accuracy of minimally invasive markers for detection of airway eosinophilia in asthma: a systematic review and meta-analysis. Lancet Respir Med 2015;3:290-300.

7. Ichinose M, Sugiura $H$, Yamagata S, Koarai A, Shirato K. Increase in reactive nitrogen species production in chronic obstructive pulmonary disease airways. Am J Respir Crit Care Med 2000;162:701-6.

8. Dweik RA, Boggs PB, Erzurum SC, Irvin CG, Leigh MW, Lundberg JO, et al. An official ATS clinical practice guideline: interpretation of exhaled nitric oxide levels (FENO) for clinical applications. Am J Respir Crit Care Med 2011;184:602-15.

9. Price DB, Buhl R, Chan A, Freeman D, Gardener E, Godley C, et al. Fractional exhaled nitric oxide as a predictor of response to inhaled corticosteroids in patients with non-specific respiratory symptoms and insignificant bronchodilator reversibility: a randomised controlled trial. Lancet Respir Med 2018;6:29-39.

10. Kimura H, Konno S, Makita H, Taniguchi N, Shimizu K, Suzuki M, et al. Prospective predictors of exacerbation status in severe asthma over a 3-year follow-up. Clin Exp Allergy 2018;48:1137-46.

11. Kimura H, Konno S, Nakamaru Y, Makita H, Taniguchi N, Shimizu K, et al. Sinus Computed Tomographic Findings in Adult Smokers and Nonsmokers with Asthma. Analysis of Clinical Indices and Biomarkers. Ann Am Thorac Soc 2017;14:332-41.

12. American Thoracic Society. Proceedings of the ATS workshop on refractory asthma: current understanding, recommendations, and unanswered questions. Am J Respir Crit Care Med 2000;162:2341-51.

13. Chung KF, Wenzel SE, Brozek JL, Bush A, Castro M, Sterk PJ, et al. International ERS/ATS guidelines on definition, evaluation and treatment of severe asthma. Eur Respir J 2014;43:343-73. 
14. Kanda Y. Investigation of the freely available easy-to-use software 'EZR' for medical statistics. Bone Marrow Transplant 2013;48:452-8.

15. Jones SL, Kittelson J, Cowan JO, Flannery EM, Hancox RJ, McLachlan CR, et al. The predictive value of exhaled nitric oxide measurements in assessing changes in asthma control. Am J Respir Crit Care Med 2001;164:738-43.

16. Roberts G, Hurley C, Bush A, Lack G. Longitudinal study of grass pollen exposure, symptoms, and exhaled nitric oxide in childhood seasonal allergic asthma. Thorax 2004;59:752-6.

17. Pijnenburg MW, Bakker EM, Hop WC, De Jongste JC. Titrating steroids on exhaled nitric oxide in children with asthma: a randomized controlled trial. Am J Respir Crit Care Med 2005;172:831-6.

18. Smith AD, Cowan JO, Brassett KP, Filsell S, McLachlan C, Monti-Sheehan G, et al. Exhaled nitric oxide: a predictor of steroid response. Am J Respir Crit Care Med 2005;172:453-9.

19. Kharitonov SA, Yates DH, Barnes PJ. Inhaled glucocorticoids decrease nitric oxide in exhaled air of asthmatic patients. Am J Respir Crit Care Med 1996;153:454-7.

20. McGrath KW, Icitovic N, Boushey HA, Lazarus SC, Sutherland ER, Chinchilli VM, et al. A large subgroup of mild-to-moderate asthma is persistently noneosinophilic. Am J Respir Crit Care Med 2012;185:612-9.

21. van der Valk RJ, Baraldi E, Stern G, Frey U, de Jongste JC. Daily exhaled nitric oxide measurements and asthma exacerbations in children. Allergy 2012;67:265-71.

22. Saito J, Gibeon D, Macedo P, Menzies-Gow A, Bhavsar PK, Chung KF. Domiciliary diurnal variation of exhaled nitric oxide fraction for asthma control. Eur Respir J 2014;43:474-84.

23. Ekroos H, Tuominen J, Sovijarvi AR. Exhaled nitric oxide and its long-term variation in healthy nonsmoking subjects. Clin Physiol 2000;20:434-9.

24. de Laurentiis G, Maniscalco M, Cianciulli F, Stanziola A, Marsico S, Lundberg JO, et al. Exhaled nitric oxide monitoring in COPD using a portable analyzer. Pulm Pharmacol Ther 2008;21:689-93.

25. Antus B, Csiszer E, Czebe K, Horvath I. Pulmonary infections increase exhaled nitric oxide in lung transplant recipients: a longitudinal study. Clin Transplant 2005;19:377-82.

26. Stern G, de Jongste J, van der Valk R, Baraldi E, Carraro S, Thamrin C, et al. Fluctuation phenotyping based on daily fraction of exhaled nitric oxide values in asthmatic children. $J$ Allergy Clin immunol 2011;128:293-300.

27. Sinha A, Lutter R, Xu B, Dekker T, Dierdorp B, Sterk PJ, et al. Loss of adaptive capacity in asthmatic patients revealed by biomarker fluctuation dynamics after rhinovirus challenge. Elife 2019;8:e47969.

28. Shome GP, Starnes JD, 3rd, Shearer M, Kennedy R, Way A, Arif A, et al. Exhaled nitric oxide in asthma: variability, relation to asthma severity, and peripheral blood lymphocyte cytokine expression. $J$ Asthma 2006;43:95-9.

\section{Tables}


. Characteristics of subjects at baseline by group, based on

; in FeNO over three years

$\begin{array}{lllll}\text { All patients } & \begin{array}{l}\text { Sustained } \\ \text { low }\end{array} & \begin{array}{l}\text { Intermediate } \\ \text { high }\end{array} & \begin{array}{l}\text { Sustained } \\ \text { value }\end{array} \\ (\mathrm{n}=100) & (\mathrm{n}=33) & (\mathrm{n}=58) & (\mathrm{n}=9) & \end{array}$

aphics

\begin{tabular}{llllll} 
ar & $58.5 \pm 12.2$ & $57.6 \pm 13.1$ & $59.9 \pm 10.8$ & $52.8 \pm 16.7$ & 0.24 \\
\hline $\mathrm{N}(\%)$ & $58(58.0 \%)$ & $18(54.6 \%)$ & $35(60.3 \%)$ & $5(55.6 \%)$ & 0.86 \\
\hline duration, year & $19.1 \pm 14.5$ & $22.0 \pm 16.3$ & $18.1 \pm 14.0$ & $15.1 \pm 9.8$ & 0.32 \\
\hline g status & $12 / 51 / 37$ & $6 / 19 / 8$ & $6 / 27 / 25$ & $0 / 5 / 4$ & 0.19 \\
t/Ex/Never) & & & & & \\
ars & $5.5(0-22.7)$ & $8.3(0.1-$ & $5.2(0-24.5)$ & $2.3(0-8.0)$ & 0.24 \\
& & $23.3)$ & & &
\end{tabular}

ass index, $\mathrm{kg} / \mathrm{m}^{2} \quad 25.5 \pm 5.0 \quad 25.3 \pm 4.6 \quad 25.3 \pm 5.3 \quad 27.0 \pm 4.7 \quad 0.63$

:S dose, $\mu \mathrm{g} \quad 1629 \pm 510 \quad 1779 \pm 548 \quad 1532 \pm 482 \quad 1700 \pm 450 \quad 0.08$

$\begin{array}{lllll}\text { lance OCS use, } \mathrm{N} \quad 37(37.0 \%) & 10(30.3 \%) & 20(34.5 \%) & 7(77.8 \%) & 0.03\end{array}$

\begin{tabular}{llllll}
\hline $\mathrm{V}(\%)$ & $61(61.0 \%)$ & $18(54.6 \%)$ & $37(63.8 \%)$ & $6(66.7 \%)$ & 0.64 \\
\hline $21.0(17.0-$ & $21.0(17.5-$ & $21.0(15.8-$ & $21.0(16.5-$ & 0.71 \\
$23.0)$ & $23.0)$ & $23.0)$ & $25.0)$ & \\
& $5.5(4.8-$ & $5.5(4.8-6.2)$ & $5.5(5.0-6.3)$ & $6.1(4.3-6.6)$ & 0.89 \\
$6.3)$ & & & &
\end{tabular}

kers

osinophil, cells/ $218(84-\quad 83(38-126) \quad 370(145-\quad 476(250-\quad<0.001$

479) 627) 577)

eutrophil, cells/ 4575 (3231- 5551 (3568- 4255 (3110- 3271 (2787- 0.19

6005) 6576) 6616) 6165) 


\begin{tabular}{llllll} 
rum IgE, IU/mL & $164(58-$ & $114(24-$ & $189(60-$ & $220(94-$ & 0.28 \\
& $402)$ & $365)$ & $419)$ & $385)$ & \\
\hline eosinophil, \% & $6.8(0.8-$ & $0.8(0.2-2.2)$ & $16.8(2.5-$ & $21.3(6.6-$ & $<0.001$ \\
& $29.6)$ & & $36.8)$ & $38.6)$ & \\
\hline spb & $27.5(17.0-$ & $15.0(10.0-$ & $37.0(24.8-$ & $91.0(74.5-$ & $<0.001$ \\
& $47.5)$ & $19.0)$ & $51.3)$ & $186.0)$ & \\
\hline ary function test & & & & & 0.98 \\
\hline o predicted & $91.6 \pm 19.2$ & $91.4 \pm 18.0$ & $91.5 \pm 19.7$ & $92.9 \pm 22.1$ & 0.98 \\
\hline VC, \% & $66.4 \pm 12.7$ & $67.3 \pm 13.9$ & $65.7 \pm 11.9$ & $67.8 \pm 14.4$ & 0.80 \\
\hline idities & & & & & 0.02 \\
\hline N (\%) & $13(13.0 \%)$ & $8(24.2 \%)$ & $3(5.2 \%)$ & $2(22.2 \%)$ & 0.40 \\
\hline rhinitis, N (\%) & $49(49.0 \%)$ & $13(39.4 \%)$ & $31(53.5 \%)$ & $5(55.6 \%)$ & 0.40 \\
\hline & $3.0(0-9.0)$ & $0(0-3.0)$ & $6.5(1.8-$ & $6.0(3.0-$ & $<0.001$ \\
& & & $12.0)$ & $12.5)$ & \\
\hline olyp, N (\%) & $25(25 \%)$ & $1(3.0 \%)$ & $21(36.2 \%)$ & $3(33.3 \%)$ & $<0.001$ \\
\hline & $8.0(3.0-$ & $8.0(4.0-$ & $8.0(3.0-$ & $3.0(1.0-$ & 0.39 \\
& $14.0)$ & $15.5)$ & $14.3)$ & $10.5)$ & \\
\hline $38.0(32.0-$ & $41.0(33.0-$ & $37.5(31.0-$ & $45.0(31.0-$ & 0.43 \\
& $46.0)$ & $47.5)$ & $45.3)$ & $54.5)$ & \\
\hline
\end{tabular}

ACT, asthma control test; AERD, aspirin-exacerbated respiratory disease; AQLQ, asthma quality of life questionnaire; FeNO, fractional exhaled nitric oxide; $\mathrm{FEV}_{1}$, forced expiratory volume in $1 \mathrm{~s}$; FVC, forced vital capacity; GERD, gastroesophageal reflux disease; ICS, inhaled corticosteroids; LMS, Lund-Mackay score; OCS, oral corticosteroids; FSSG, frequency scale for the symptoms of GERD; SDS, self-reported depression scale. Data are shown as the mean \pm standard deviation, median (interquartile range), or number (\%). 
P-values were obtained using a chi-square test, one-way analysis of variance, or the Kruskal-Wallis test, as appropriate. 
Characteristics of subjects in the low CV and high CV intermediate groups at

$\begin{array}{lll}\text { Low CV } & \text { High CV } & \text { P-value } \\ (\mathrm{n}=29) & (\mathrm{n}=29) & \end{array}$

\section{aphics}

\begin{tabular}{llll}
\hline ar & $61.1 \pm 12.6$ & $58.7 \pm 8.8$ & 0.39 \\
\hline $\mathrm{N}(\%)$ & $20(69.0 \%)$ & $15(51.7 \%)$ & 0.18 \\
\hline duration, year & $21 \pm 16.0$ & $15.2 \pm 11.2$ & 0.11 \\
\hline g status (Current/Ex/Never) & $4 / 9 / 16$ & $2 / 18 / 9$ & 0.06 \\
\hline ars & $0(0-16.0)$ & $11.0(0-37.5)$ & 0.04 \\
\hline ass index, $\mathrm{kg} / \mathrm{m}^{2}$ & $24.6 \pm 3.6$ & $26.1 \pm 6.6$ & 0.31 \\
\hline :S dose, $\mu \mathrm{g}$ & $1412 \pm 444$ & $1653 \pm 495$ & 0.06 \\
\hline lance OCS use, $\mathrm{N}(\%)$ & $9(31.0 \%)$ & $11(37.9 \%)$ & 0.58 \\
\hline $\mathrm{v}(\%)$ & $15(51.7 \%)$ & $22(75.9 \%)$ & 0.05 \\
\hline & $21.0(18.5-23.0)$ & $20.0(14.5-23.0)$ & 0.20 \\
\hline & $5.6(5.2-6.5)$ & $5.4(4.7-6.1)$ & 0.14
\end{tabular}

kers

osinophil, cells $/ \mu \mathrm{L}$

389 (145-593)

332 (144-678)

0.69

eutrophil, cells/pL

4522 (3182-6096)

$3994(3078-5496)$

0.39

rum IgE, IU/mL

170 (59-378)

$194(81-501)$

0.67

eosinophil, \%

$14.2(2.0-27.9)$

$27.6(4.0-38.8)$

0.31

spb

$31.0(24.0-43.0)$

42.0 (28.0-64.5)

0.19

ary function test

ó predicted

$94.1 \pm 18.7$

$88.2 \pm 20.7$

0.26

VC, \%

$65.6 \pm 12$

$65 \pm 12.6$

0.84

idities

$\%)$

$2(6.9 \%)$

$1(3.5 \%)$

0.55 


\begin{tabular}{llll} 
rhinitis, N (\%) & $15(51.7 \%)$ & $16(55.2 \%)$ & 0.79 \\
\hline olyp, N (\%) & $7.0(2.0-10.0)$ & $6.0(1.0-12.0)$ & 0.80 \\
\hline & $12(41.4 \%)$ & $9(31.0 \%)$ & 0.41 \\
\hline $7(1-14)$ & $9(6-15.5)$ & 0.07 \\
\hline
\end{tabular}

$\mathrm{ACT}$, asthma control test; AERD, aspirin-exacerbated respiratory disease; AQLQ, asthma quality of life questionnaire; FeNO, fractional exhaled nitric oxide; $\mathrm{FEV}_{1}$, forced expiratory volume in 1 s; FVC, forced vital capacity; GERD, gastroesophageal reflux disease; ICS, inhaled corticosteroids; LMS, Lund-Mackay score; OCS, oral corticosteroids; FSSG, frequency scale for the symptoms of GERD; SDS, self-reported depression scale.

Data are shown as the mean \pm standard deviation, median (interquartile range), or number (\%).

P-values were obtained using a chi-square test, student's t-test, or the Mann-Whitney $U$ test, as appropriate.

Table 3. Factors associated with exacerbations in the intermediate group

Exacerbation-free survival

\begin{tabular}{lll}
\hline Variable & Hazard ratio (95\% CI) & P-value \\
\hline CV of FeNO & $6.01(1.09-29.29)$ & 0.04 \\
\hline
\end{tabular}

Exacerbation frequency

\begin{tabular}{lll}
\hline Variable & Rate ratio (95\% CI) & P-value \\
\hline CV of FeNO & $7.76(2.32-26.15)$ & 0.001 \\
\hline
\end{tabular}

$\mathrm{CV}$, coefficient of variation; FeNO, fractional exhaled nitric oxide.

The hazard ratio was derived from the multivariate Cox proportional hazards model, and the rate ratio was derived from the multivariate Poisson regression model. Both models 
were adjusted for age, sex, body mass index, pack-years, blood eosinophil count, and FeNO at baseline.

Figures

Figure 1

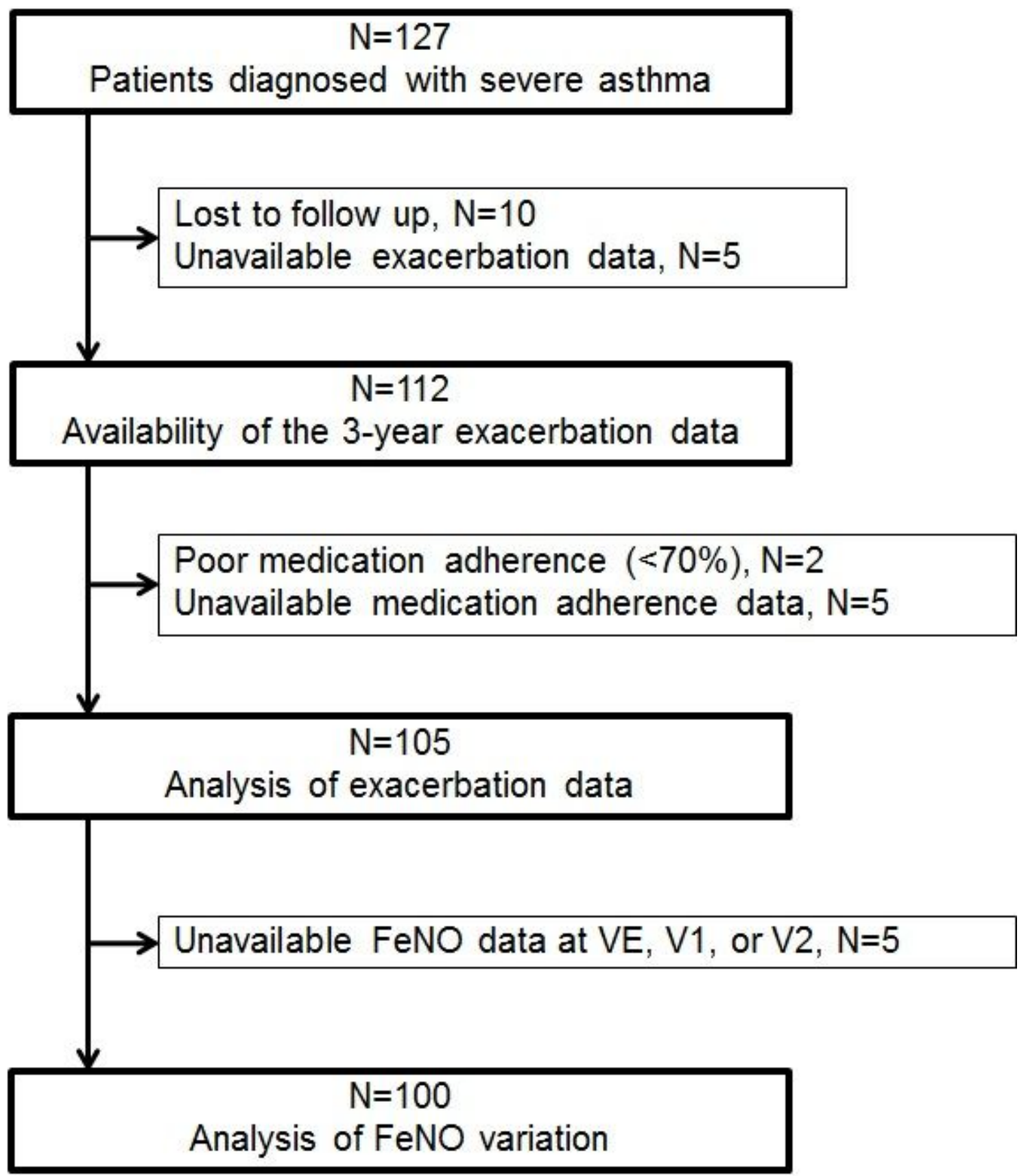

Figure 1 
Figure 2

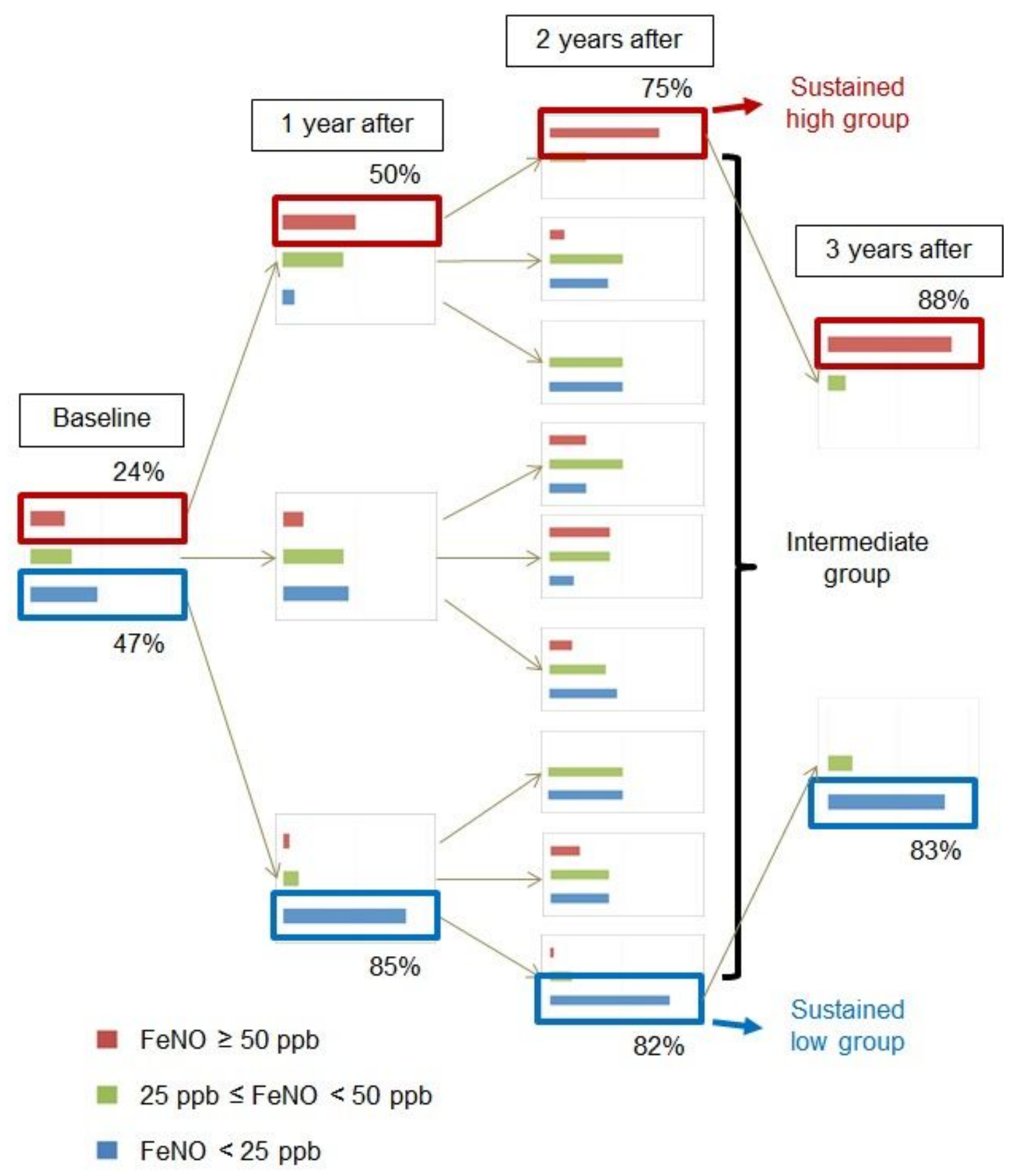

Figure 2

Changes in FeNO over a 3-year follow-up period 
Figure 3

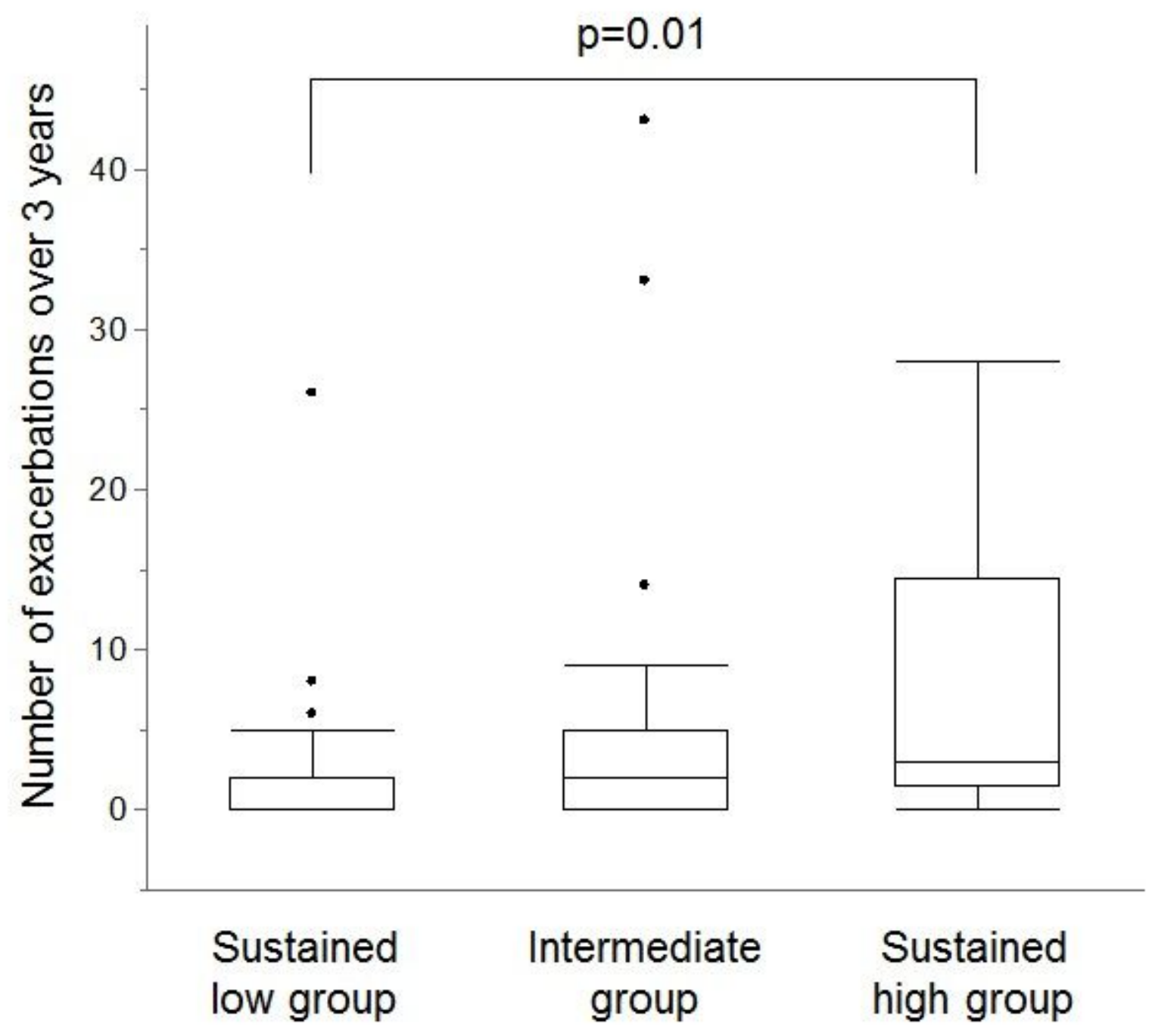

Figure 3

Box plots of exacerbation frequencies over 3 years The $p$-values were obtained using the Kruskal-Wallis test with a post-hoc Dunn's multiple comparison test. 
Figure 4

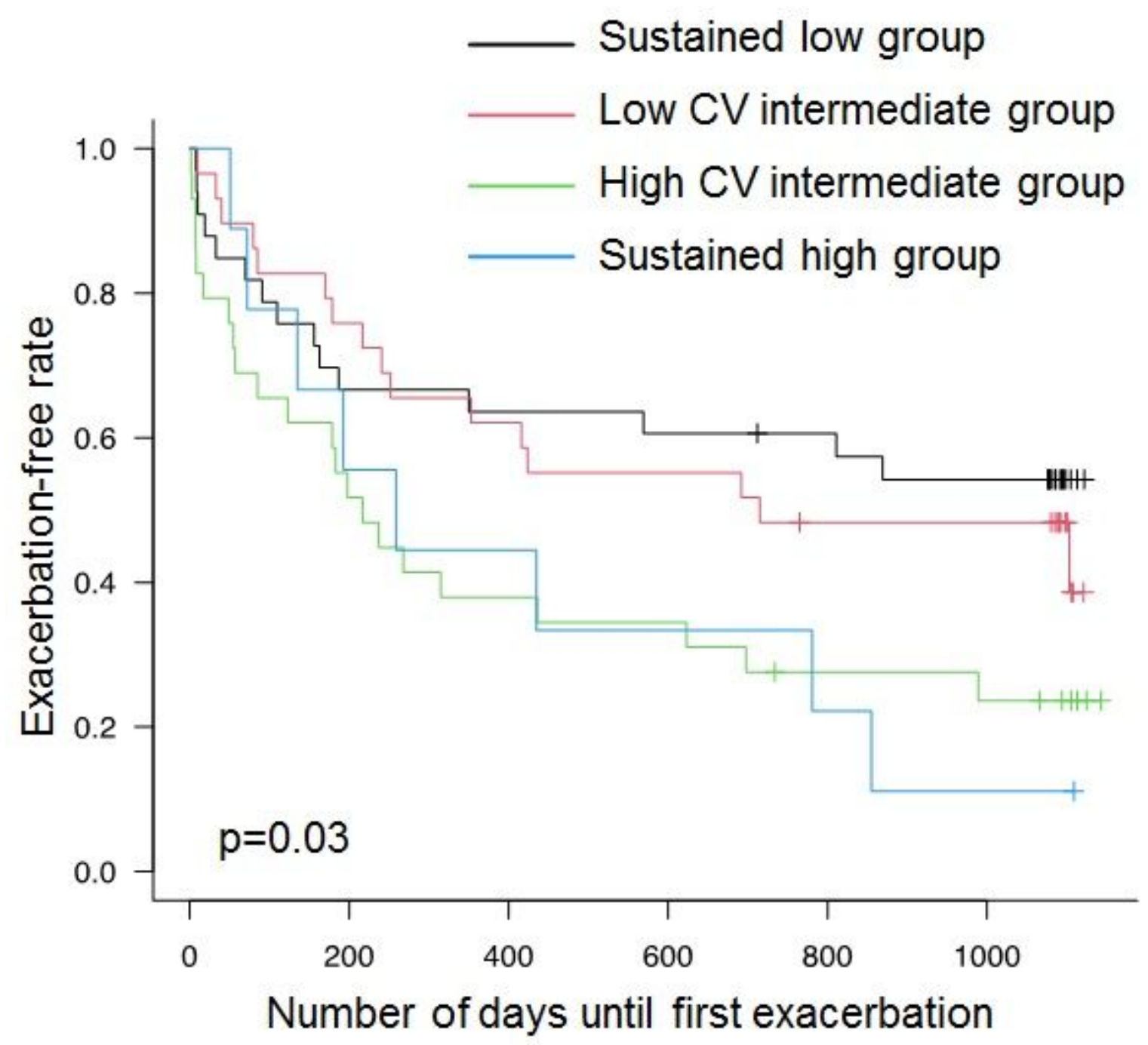

Figure 4

Kaplan-Meier curves for exacerbation-free survival The intermediate group was divided into two groups, and four groups were compared. 
Figure 5

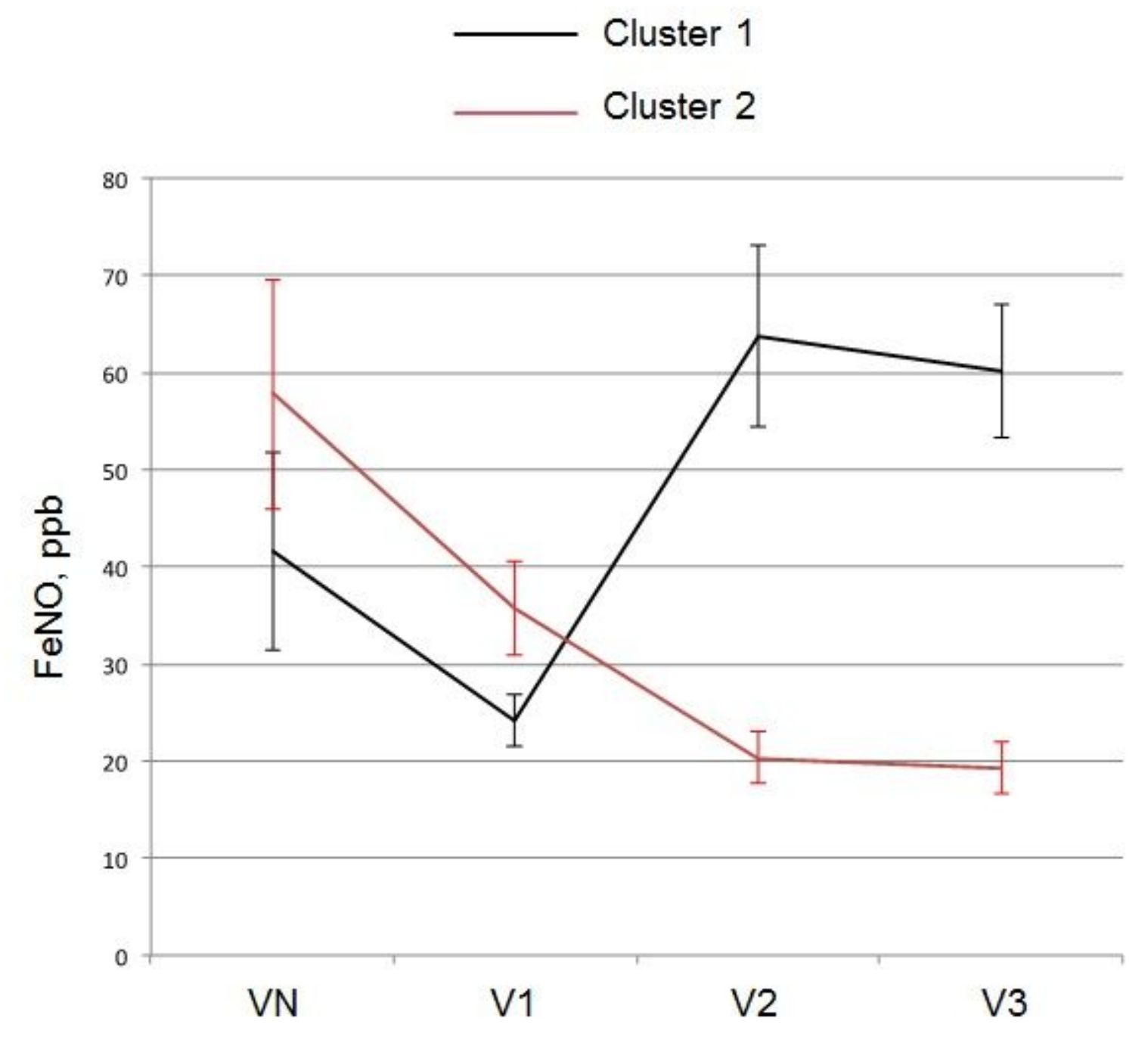

Figure 5

Annual changes in FeNO of the high CV intermediate group The high CV intermediate group was divided into two groups by cluster analysis. Data are shown as the mean \pm standard error. 
Figure 6

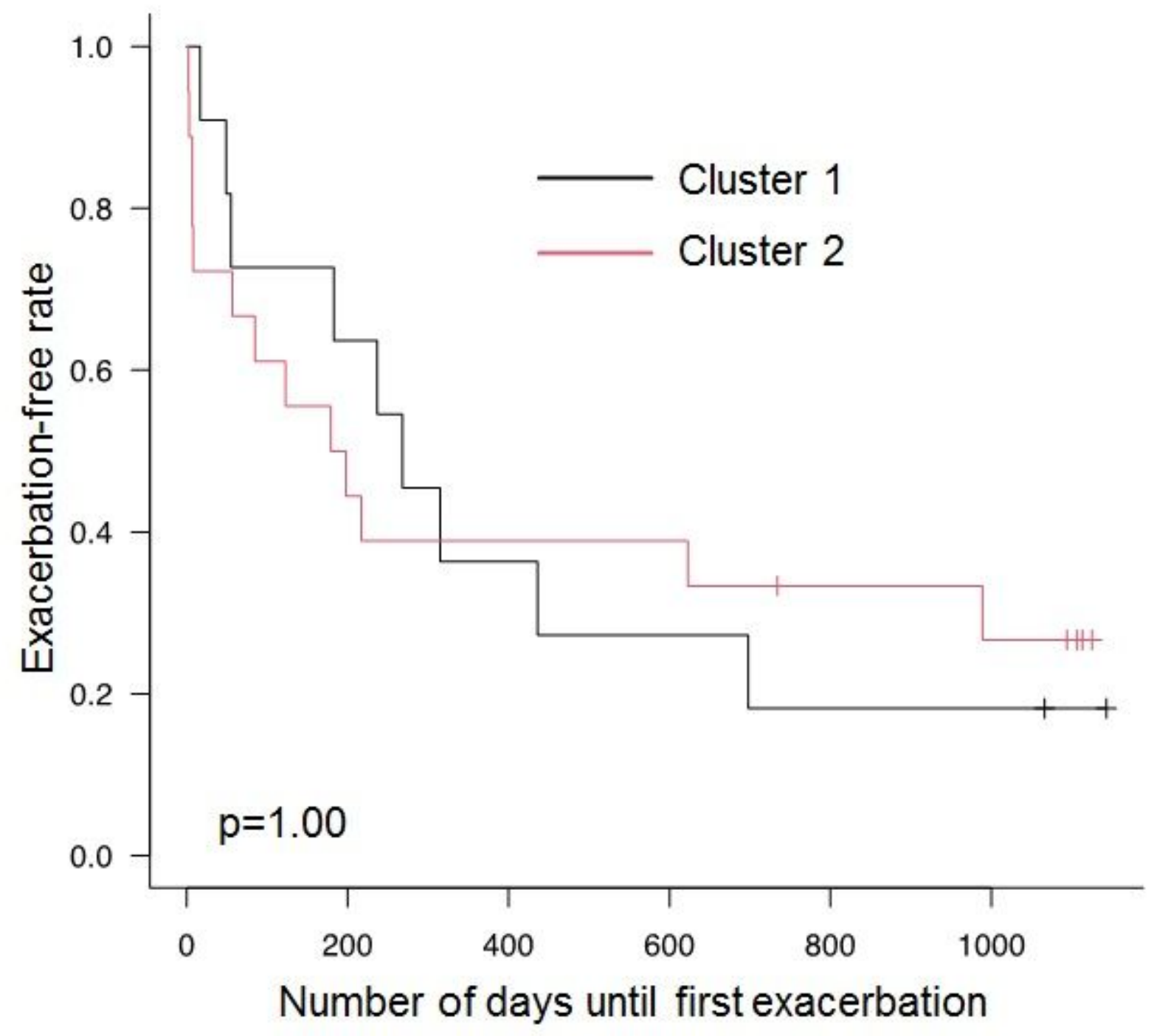

Figure 6

Kaplan-Meier curves for exacerbation-free survival The high CV intermediate group was divided into two groups by cluster analysis.

\section{Supplementary Files}

This is a list of supplementary files associated with this preprint. Click to download.

- STROBEChecklist.docx 